\title{
Avaliação clínica de crianças e adolescentes asmáticos: relevância da atenção interdisciplinar
}

\section{Clinical evaluation of asthmatic children and adolescents: relevance of interdisciplinary attention}

Karina Machado Siqueira ${ }^{1}$, Geovanna Líscio Pereira ${ }^{2}$, André Luiz Bittencourt ${ }^{3}$, Camila Silva Colodino ${ }^{4}$, Isabela Cristine Ferreira Fernandes ${ }^{5}$, Maria Alves Barbosa ${ }^{6}$

\footnotetext{
${ }^{1}$ Enfermeira. Doutora em Enfermagem. Professora Adjunta da Faculdade de Enfermagem (FEN) da Universidade Federal de Goiás (UFG). Goiânia, GO, Brasil. E-mail: karinams.fen@gmail.com.

${ }^{2}$ Enfermeira. Discente do Programa de Residência Multiprofissional em Saúde, área Materno Infantil, do Hospital das Clínicas da UFG. Goiânia, GO, Brasil. Email: geovanna_liscio@hotmail.com.

${ }^{3}$ Enfermeiro. Enfermeira da Empresa Sempre Saúde. Goiânia, GO, Brasil. E-mail: bitencourtandre@hotmail.com.

${ }^{4}$ Enfermeira. Discente do Programa de Residência em Enfermagem Oncológica da Associação de Combate ao Câncer em Goiás. Goiânia, Go, Brasil. E-mail: mila.silva6@hotmail.com.

${ }^{5}$ Enfermeira, Mestre em Enfermagem. Discente do Programa de Pós-Graduação em Enfermagem, nível Doutorado, FEN/UFG. Professora Assistente da Universidade Federal de Tocantins. Palmas, TO, Brasil. E-mail: isabela_cristine@hotmail.com.

${ }^{6}$ Enfermeira. Doutora em Enfermagem. Professora Associada da FEN/UFG. Goiânia, GO, Brasil. E-mail: maria.malves@gmail.com.
}

\section{RESUMO}

Estudo descritivo, retrospectivo, que objetivou avaliar as condições clínicas de crianças e adolescentes com asma, ao iniciarem o tratamento e após seis meses de acompanhamento por equipe multiprofissional, com foco na atenção interdisciplinar. Pesquisa desenvolvida em ambulatório de referência terciária para a atenção à asma infantil, em Goiânia, Goiás, Brasil. Foram coletados dados de 101 prontuários entre janeiro e março de 2013. As crianças e adolescentes eram predominantemente do sexo masculino (70,3\%), com idade entre dois e cinco anos (31,7\%) e classificadas como casos de asma persistente moderada (47,6\%). Após o acompanhamento durante seis meses, verificou-se que a maioria $(64,4 \%)$ obteve controle total ou parcial da doença, redução de todos os sintomas identificados na primeira consulta e apresentaram alta adesão ao tratamento farmacológico proposto (70,3\%). A atenção interdisciplinar pode resultar na melhoria do manejo clínico e contribuir para o avanço na qualidade da assistência a crianças e adolescentes asmáticos.

Descritores: Asma; Criança; Adolescente; Assistência Integral à Saúde; Enfermagem Pediátrica.

\section{ABSTRACT}

Descriptive, retrospective study, aimed to evaluate clinical conditions of asthmatic children and adolescents at the beginning of treatment and after six months of accompaniment by a multi-professional team, focused on interdisciplinary attention. A research developed in a reference ambulatory for tertiary attention of infant asthma, in Goiânia, Goiás, Brazil. Data was collected from 101 medical records from January and March 2013. Children and adolescents were predominantly male $(70,3 \%)$, aged between two and five years $(31,7 \%)$ and clinically classified as moderated persistent asthma (47,6\%). After following them for six months, it was seen that most $(64,4 \%)$ obtained total or partial control of the disease, reduction of all symptoms identified in the first consultation and presented high adherence to the proposed pharmacological treatment $(70,3 \%)$. Interdisciplinary attention can result in improved clinical control and contribute to advances in assistance quality to asthmatic children and adolescents.

Descriptors: Asthma; Child; Adolescent; Comprehensive Health Care; Pediatric Nursing. 


\section{INTRODUÇÃO}

As doenças crônicas, em destaque a asma, podem acometer a saúde infantil e remeter a uma adaptação e modificação de hábitos de vida, causando um profundo impacto na criança, no adolescente e em suas famílias.

A asma é considerada a doença respiratória crônica mais prevalente em crianças e adolescentes e caracterizase pela hiper-responsividade das vias aéreas que levam a episódios recorrentes de sibilos, dispneia, opressão torácica e tosse, particularmente, à noite ou no início da manhã. Esses episódios são uma consequência da obstrução do fluxo aéreo intrapulmonar, generalizada e variável, reversível espontaneamente ou com tratamento ${ }^{(1)}$.

É uma condição potencialmente grave, cuja prevalência tem aumentado em todo o mundo, sendo um problema mundial de saúde ${ }^{(1-2)}$. Nas últimas décadas, apesar dos avanços no entendimento da fisiopatogenia e no tratamento, sua morbidade e mortalidade têm crescido em todo o mundo ${ }^{(3)}$. Em 2011 foram registradas pelo DATASUS 160.000 hospitalizações, dado que colocou a asma como a quarta causa de internações ${ }^{(4)}$. A taxa média de mortalidade no país, entre 1998 e 2007, foi de 1,52/100.000 habitantes (variação, 0,85-1,72/100.000 habitantes), com estabilidade na tendência temporal desse período ${ }^{(1)}$.

Doença que gera encargos significativos em termos de custos em saúde, que aumenta proporcionalmente com a sua gravidade, também gera prejuízos importantes na dinâmica social, especialmente no que se refere às perdas de produtividade, menor participação na vida familiar e risco de exclusão social ${ }^{(1-2,5)}$.

Diante da relevância epidemiológica e social dessa doença, as discussões sobre a atenção aos portadores de asma têm sido expandidas. No Brasil, em 1999, foram iniciadas as discussões no campo das políticas públicas e criou-se o Plano Nacional de Controle da Asma - PNCA. Partindo das orientações desse plano, diversos programas de controle e atenção à asma foram criados e consolidados no país ${ }^{(5-6)}$.
Por se tratar de uma condição crônica, que necessita de tratamento complexo e prolongado, crianças e adolescentes com asma necessitam de um adequado acompanhamento por equipe multiprofissional e do envolvimento de seus cuidadores. Para o controle da asma, além do tratamento farmacológico adequado, é necessário que a criança, o adolescente e sua família tenham conhecimento sobre a doença, os fatores desencadeantes, os sinais e sintomas das exacerbações, além da segurança no uso de medicações ${ }^{(7-8)}$.

A asma, portanto, tem impacto importante na vida dos pacientes, seus familiares e no sistema de saúde. Embora não exista cura, o manejo adequado baseado na parceria profissional-paciente-família pode resultar em controle da doença ${ }^{(1,3)}$. Apesar de as questões relacionadas à fisiopatologia da doença, fatores desencadeantes e tratamento farmacológico, já estarem bem estabelecidas e discutidas entre os especialistas, os aspectos relativos à atenção multiprofissional e os resultados do trabalho interdisciplinar, voltado ao cuidado de crianças e adolescentes asmáticos, ainda precisam ser melhor conhecidos.

Nesse contexto, ressalta-se a importância dos profissionais de saúde na atenção interdisciplinar voltada a essas crianças e adolescentes. Interdisciplinaridade entendida aqui como ações integradas e interrelacionadas de diferentes profissionais, que buscam instaurar a totalidade em um campo de saber complexo, múltiplo e heterogêneo ${ }^{(9)}$. Essa ação integrada, baseada no saber de diferentes disciplinas, pode colaborar no enfrentamento da doença e se tornar fundamental para que paciente e cuidador consigam atingir adequado e efetivo nível de conscientização e mudança de comportamento frente à sua problemática.

Diante destas considerações, este estudo objetivou avaliar as condições clínicas de crianças e adolescentes com asma antes e após seis meses de acompanhamento multiprofissional, baseado na atenção interdisciplinar, e promovido em ambulatório de referência terciária, especializado em asma infantil. 
A condução desse estudo se justifica pela possibilidade de agregar novos conhecimentos e ampliar a discussão a respeito da atenção interdisciplinar no cuidado a crianças e adolescentes asmáticos. O diálogo contínuo entre diferentes áreas do conhecimento, de maneira compartilhada e interativa, poderá facilitar os enfrentamentos profissionais e a promoção de uma assistência humanizada, contribuindo para a avaliação das ações existentes e o estabelecimento de novas estratégias de atenção multiprofissional qualificada no contexto da asma infantil.

\section{METODOLOGIA}

Estudo retrospectivo, descritivo e quantitativo, do tipo documental, realizado por meio da análise das informações disponíveis nos prontuários de crianças e adolescentes atendidos em um ambulatório de referência para o tratamento da asma infantil, localizado em um hospital de grande porte, do município de Goiânia-Goiás, Brasil. O serviço integra a rede de cuidados terciários do Catavento - Programa de Controle da Asma de Goiânia e também oferece atendimento aos demais municípios do estado de Goiás. A opção em eleger esse local atribui-se ao seu reconhecimento e referência em oferecer atenção interdisciplinar às crianças e adolescentes asmáticos.

A atenção interdisciplinar do referido ambulatório acontece por meio de ações integradas entre profissionais de diferentes áreas do conhecimento. Estão envolvidos na equipe, profissionais, residentes e acadêmicos de enfermagem, fonoaudiologia, medicina, nutrição e serviço social. São realizadas consultas individuais que procuram atender à saúde integral das crianças, adolescentes e seus cuidadores, bem como, atividades grupais de educação em saúde. Apesar de suas especificidades, todos os profissionais estão atentos aos diversos aspectos físicos, emocionais, sociais e ambientais, que podem, direta ou indiretamente, influenciar no manejo e convívio com a asma. As ações são integradas e discutidas entre os diversos membros da equipe multiprofissional, que buscam sempre evitar a fragmentação do cuidado, valorizando e respeitando as contribuições de cada área profissional. A interdisciplinaridade faz-se presente nesta integração e na intensidade de trocas e comunicação entre os profissionais.

Os prontuários de crianças e adolescentes com asma foram incluídos considerando-se aquelas de idade entre zero e 18 anos, que iniciaram acompanhamento por equipe multiprofissional, entre os meses de janeiro de 2010 e julho de 2012, e continuaram em atendimento no mesmo serviço durante os seis meses seguintes à primeira consulta. Assim, foram solicitados 141 prontuários referentes ao período proposto, sendo localizados apenas 119 pelo serviço de arquivo da instituição. Após a análise, 18 prontuários foram excluídos, devido à inexistência de ficha constando registro do retorno após seis meses de acompanhamento. Assim, neste estudo foram incluídos 101 prontuários.

A coleta de dados foi realizada no período entre janeiro e março de 2013, por três auxiliares de pesquisa devidamente treinados. Todos os aspectos éticos foram observados seguindo-se a legislação brasileira ${ }^{(10)}$. estudo foi submetido ao Comitê de Ética em Pesquisa Médica Humana e Animal do Hospital das Clínicas da Universidade Federal de Goiás - CEP/HC/UFG e aprovado sob o número de parecer 119/2010.

Os dados foram coletados em instrumento que contemplava aspectos demográficos e clínicos das crianças e adolescentes, incluindo classificação da asma, sintomas, tratamento farmacológico e nível de controle da doença. Todas as informações estavam contidas nas fichas de consulta padronizadas pelo serviço e preenchidas pela equipe multiprofissional responsável pelo atendimento. As fichas selecionadas para coleta de dados eram referentes ao primeiro atendimento e ao retorno após seis meses de acompanhamento.

As fichas de consulta, utilizadas pelos profissionais durante os atendimentos do ambulatório e arquivadas nos prontuários, apresentavam informações sobre a saúde integral da criança ou adolescente, bem como, 
dados específicos sobre o diagnóstico e manejo da asma, os quais constam em recomendações de diretrizes nacionais e internacionais ${ }^{(1-3)}$.

A análise dos dados do prontuário foi feita por duas pesquisadoras, de forma independente. A entrada e a edição dos dados foram efetuadas com o auxílio de um software e analisadas de forma descritiva.

\section{RESULTADOS}

A análise dos registros realizados pela equipe multiprofissional durante o primeiro atendimento às crianças e adolescentes, possibilitou identificar características demográficas e a classificação da asma no início do acompanhamento no ambulatório especializado.

A população do estudo foi composta predominantemente pelo sexo masculino $(70,3 \%)$, com idade entre dois e cinco anos $(31,7 \%)$, procedente de cidades do interior do Estado de Goiás (55,5\%) e classificada como casos de asma persistente moderada (47,6\%), conforme demonstra a Tabela 1.

Tabela 1: Características demográficas e clínicas de crianças e adolescentes com asma ao iniciarem acompanhamento ambulatorial especializado entre os meses de janeiro de 2010 e julho de 2012. Goiânia, GO, Brasil, 2013.

\begin{tabular}{|c|c|c|}
\hline Características demográficas e clínicas & $N=101$ & $\%$ \\
\hline \multicolumn{3}{|l|}{ Sexo } \\
\hline Masculino & 71 & 70,3 \\
\hline Feminino & 30 & 29,7 \\
\hline \multicolumn{3}{|l|}{ Idade } \\
\hline$<$ Dois anos & 10 & 9,9 \\
\hline Dois a cinco anos & 32 & 31,7 \\
\hline Seis a nove anos & 30 & 29,7 \\
\hline $10-15$ anos & 29 & 28,7 \\
\hline \multicolumn{3}{|l|}{ Procedência } \\
\hline Capital & 37 & 36,6 \\
\hline Cidades do interior & 56 & 55,5 \\
\hline Não informado & 08 & 7,9 \\
\hline \multicolumn{3}{|l|}{ Classificação da Asma } \\
\hline Lactente sibilante & 09 & 8,9 \\
\hline Intermitente & 06 & 5,9 \\
\hline Persistente leve & 09 & 8,9 \\
\hline Persistente moderada & 48 & 47,6 \\
\hline Persistente grave & 10 & 9,9 \\
\hline Não informado & 19 & 18,8 \\
\hline
\end{tabular}

Vale ressaltar que, apesar de o ambulatório em estudo, atender adolescentes até 18 anos de idade, a frequência de pacientes que iniciaram o tratamento com idade superior a 15 anos foi nula durante o período do estudo.

Segundo os registros das fichas de primeiro atendimento, verificou-se que $78(77,2 \%)$ necessitaram de atendimento em serviços de urgência/emergência, mediante a situação de exacerbação da asma, e dentre esses, 74 (73,3\%) foram hospitalizados devido à crise asmática. No entanto, somente $33(32,7 \%)$ crianças e adolescentes haviam realizado algum tratamento para asma antes de serem encaminhados ao serviço de referência.

Em relação aos sintomas observados, constatou-se que os mais frequentes foram: tosse, dispneia, sibilância, aperto torácico, gotejamento pós-nasal, espirros, prurido nasal, prurido ocular e coriza aquosa. A Tabela 2 apresenta a frequência dos sintomas identificados no primeiro atendimento e no retorno após seis meses. 
Tabela 2. Sintomas apresentados por crianças e adolescentes com asma durante o primeiro atendimento e após seis meses de acompanhamento multiprofissional em serviço especializado. Goiânia, GO, Brasil, 2013.

\begin{tabular}{|c|c|c|c|c|}
\hline \multirow{2}{*}{ Sintomas } & \multicolumn{2}{|c|}{ Primeiro atendimento } & \multicolumn{2}{|c|}{ Retorno (após seis meses) } \\
\hline & $\mathrm{N}=101$ & $\%$ & $\mathrm{~N}=101$ & $\%$ \\
\hline Tosse & 71 & 70,3 & 48 & 47,5 \\
\hline Dispneia & 75 & 74,2 & 38 & 37,6 \\
\hline Sibilância & 53 & 52,5 & 17 & 16,8 \\
\hline Aperto no peito & 04 & 3,96 & 01 & 0,9 \\
\hline Gotejamento pós-nasal & 12 & 11,9 & 0,0 & 0,0 \\
\hline Espirros & 66 & 65,3 & 17 & 16,8 \\
\hline Prurido nasal & 64 & 63,3 & 18 & 17,8 \\
\hline Coriza aquosa & 59 & 58,4 & 15 & 14,8 \\
\hline Prurido ocular & 41 & 40,5 & 04 & 3,9 \\
\hline
\end{tabular}

Após seis meses de acompanhamento multiprofissional, verificou-se que $57(56,4 \%)$ crianças e adolescentes apresentaram pelo menos uma crise asmática, 30 (29,7\%) não apresentaram e 14 (13,9\%) não souberam informar, pois não identificaram os sintomas da crise asmática. Dentre aqueles que tiveram crises ou exacerbações da doença, identificou-se que a maioria $(59,6 \%)$ conseguiu relacionar a crise a algum evento ocorrido.

Um dos aspectos avaliados nos atendimentos de retorno das crianças e adolescentes, após seis meses de tratamento, foi o nível de controle atual da asma. Dentre os casos estudados, a maioria apresentou algum controle da doença após o período de acompanhamento, sendo 22 casos $(21,8 \%)$ com asma totalmente controlada, 43 $(42,6 \%)$ asma parcialmente controlada, seis $(5,9 \%)$ asma não controlada. Entretanto, em 29 casos (28,7\%) não havia registro dessa avaliação.

No que diz respeito à adesão ao tratamento farmacológico, identificou-se que, após seis meses de tratamento, $71(70,3 \%)$ crianças e adolescentes estavam em uso de alguma medicação específica para o tratamento da asma, seguindo as orientações recomendadas pelos profissionais.

Dentre os que aderiram ao tratamento com medicação específica, a via inalatória foi a mais utilizada e verificou-se que 51 (71,8\%) estavam em uso de inalador pressurizado, também conhecido como aerossol dosimetrado ou spray. Além deste, também foi registrado o uso do inalador de pó seco em 14 casos $(19,7 \%)$, onde o medicamento é apresentado em cápsulas para inalação. Somente seis $(8,5 \%)$ crianças e adolescentes usavam medicamento em outra forma de apresentação.

Dentre as crianças e adolescentes que relataram fazer uso de medicamentos apresentados no formato de inalador pressurizado, 97 (96\%) indicaram utilizar espaçadores acoplados ao dispositivo.

\section{DISCUSSÃO}

A asma é considerada um problema de saúde mundial. O impacto na vida dos pacientes e familiares, bem como nos sistemas de saúde é evidente. Estudos acerca dessa temática demonstram a importância de um cuidado integral a crianças e adolescentes asmáticos ${ }^{(1,3,6)}$. No entanto, adotar uma abordagem interdisciplinar para o atendimento ao paciente, com a finalidade de alcançar melhores resultados no que se refere ao manejo da doença e resposta clínica do asmático, consiste em um importante desafio para os profissionais que atuam nessa área.

Os serviços de atenção a essa população precisam estar preparados para receber suas demandas e o modo de cuidado pode refletir significamente na resposta clínica esperada.

As doenças crônicas, em especial a asma, são de tratamento complexo, exigindo participação ativa do próprio asmático e de seus cuidadores. A cronicidade remete a uma adaptação e modificação de hábitos de vida, causando um profundo impacto no asmático, em sua família e na sociedade ${ }^{(7,11)}$. O não controle do agravo 
leva ao uso recorrente dos serviços de emergência e a necessidade frequente de internações geram um grande ônus, tanto para as crianças, com perda de dias de escola, como para os pais, com faltas no serviço ${ }^{(3,7-8)}$.

A maior incidência da asma em crianças e adolescentes do sexo masculino é esperado, por considerar um menor tamanho das vias aéreas neste grupo ao nascer ${ }^{(12)}$. Já na idade adulta os números se invertem e a incidência é maior entre mulheres ${ }^{(12-13)}$.

Os sintomas da asma, na maioria dos casos, se apresentam precocemente e a procura pelo serviço especializado ocorre durante as primeiras apresentações clínicas da doença. Isso reforça o fato do uso recorrente de serviços especializados e de urgência ser maior entre crianças do que entre adolescentes ${ }^{(3,14)}$.

Importante destacar que a identificação dos sintomas e correlação precoce com a asma pode possibilitar intervenções mais efetivas, evitando complicações relacionadas à doença. Deste modo, profissionais envolvidos na atenção à saúde da criança, em todos os níveis de complexidade, precisam estar atentos aos sintomas que caracterizam a asma, oferecendo assistência adequada ou referenciando aos serviços especializados.

Dentre as 101 crianças e adolescentes, a maioria $(55,5 \%)$ é proveniente de cidades do interior do estado de Goiás. Esse fato ressalta a importância do atendimento realizado no ambulatório especializado, pois se trata de referência regional. No entanto, torna-se imprescindível a criação e ampliação de programas que visem o tratamento da asma e a capacitação dos profissionais que atuam nas cidades do interior, poupando o deslocamento desses pacientes e a sobrecarga dos serviços nas capitais.

A classificação da asma é uma conduta importante no manejo da doença. Considera-se a frequência das crises asmáticas, a sintomatologia, o tratamento atual, o histórico e os exames clínicos ${ }^{(15-16)}$. Fatores esses que contribuem para uma melhor precisão da classificação, possibilitando intervenções resolutivas por meio de esquemas terapêuticos mais eficientes, além de ser útil como parâmetro comparativo da avaliação do plano de tratamento proposto ${ }^{(1,12)}$.

A realização de tratamento é um outro parâmetro importante na avaliação da doença. Percebe-se que poucas crianças e adolescentes são tratadas com algum medicamento específico ou de uso contínuo antes de serem encaminhadas a serviços de referência ${ }^{(5,8)}$. Isso demonstra que os asmáticos comumente são tratados somente durante a exacerbação dos sintomas. O contato com o profissional de saúde acaba acontecendo, geralmente, apenas nos serviços de urgência/emergência, em que a situação de crise asmática é crítica e a hospitalização pode ser necessária para amenizar os sintomas.

Nesse contexto, importante salientar o papel dos profissionais de saúde, na atenção básica ${ }^{(17)}$, que precisam estar habilitados para identificar, orientar e acompanhar os pacientes e seus cuidadores. Essas ações multiprofissionais englobam desde o reconhecimento dos casos até a conscientização do impacto da asma enquanto doença crônica na infância e adolescência.

A educação em saúde torna-se imprescindível para que os asmáticos e seus familiares compreendam a necessidade de relacionar e identificar os fatores desencadeantes da crise, uso correto da medicação e os sinais e sintomas. O reconhecimento da sintomatologia de uma crise asmática, caracterizada por dispneia expiratória intensa e sibilos, é fundamental para que as intervenções sejam resolutivas ${ }^{(1,16)}$.

Os principais sintomas apresentados por crianças e adolescentes asmáticos durante atendimento em serviço especializado foram tosse, dispneia e sibilância ${ }^{(1,13,18)}$. Importante ressaltar que, para o manejo da asma, são primordiais a identificação dos sintomas e dos fatores desencadeantes, bem como, a adesão e o uso correto das medicações $^{(1,3,18)}$.

Apesar de $56,4 \%$ das crianças e adolescentes terem apresentado alguma crise asmática após seis meses de acompanhamento, na avaliação de controle atual da asma, a maioria $(64,4 \%)$ apresentou asma totalmente ou 
parcialmente controlada. No serviço em estudo, a avaliação de controle atual da asma é feita utilizando-se informações relativas às últimas quatro semanas, segundo o Asthma Control Test - $\mathrm{ACT}^{(16)}$.

Outro fato relevante foi que para maioria das crianças ou adolescentes que apresentaram crises $(59,6 \%)$, o próprio paciente ou familiar conseguiu relatar os fatores desencadeantes da crise. O desenvolvimento desta habilidade de identificar fatores que exacerbam os sintomas é essencial para o manejo da doença ${ }^{(2-3)}$. A exacerbação da doença pode estar relacionada à dificuldade financeira em adquirir as medicações, adaptar o ambiente domiciliar, ou ainda pelo contato frequente com familiar tabagista ${ }^{(19-20)}$.

Além do controle atual da asma, a adesão à terapêutica medicamentosa específica para a doença foi algo que se destacou no presente estudo, pois a maioria das crianças e adolescentes (70,3\%) estava em uso de algum medicamento após seis meses de acompanhamento e a via inalatória foi a mais utilizada.

O tratamento medicamentoso da asma é feito através dos medicamentos de controle e alívio. Os medicamentos tidos como controladores são os que apresentam ação anti-inflamatória, os corticosteroides inalatórios e, como medicamentos de alívio, os broncodilatadores. A escolha do uso individual ou associado está relacionada à gravidade da doença ${ }^{(3,7-8)}$.

Os medicamentos inalatórios são mais eficientes, pois são lançados diretamente nas vias aéreas, reduzindo perdas, aumentando a concentração local da droga e diminuindo efeitos colaterais sistêmicos. Entretanto, o uso incorreto desses dispositivos concorre para o insucesso da terapêutica ${ }^{(21-22)}$.

Dentre as causas para o uso incorreto, verifica-se o uso do aerossol dosimetrado diretamente na boca. Esse tipo de medicação deve ser usada juntamente com espaçadores, a fim de se ter uma maior eficácia da medicação ${ }^{(23)}$. Durante as crises ou exacerbações, o broncodilatador acoplado a espaçador, fornece uma reversão mais rápida do broncoespasmo induzido na crise asmática do que aquela conseguida por meio do inalador de pó seco ${ }^{(22-23)}$.

Nesse contexto, a equipe multiprofissional pode atuar em colaboração com os pacientes e familiares no sentido de estabelecer a efetividade do tratamento farmacológico e não farmacológico da doença ${ }^{(3,7,18,22)}$.

No local onde foi desenvolvido este estudo, a atuação profissional propõe a superação de um modelo que privilegia o trabalho individual de uma categoria profissional, promovendo o planejamento, discussão e execução de ações compartilhadas. Desse modo, a atenção interdisciplinar constitui em uma ação permanente, que parece ser impactante para as crianças e adolescentes asmáticos e seus familiares.

A interdisciplinaridade é uma colaboração que precisar ser propagada para melhorar a integração e comunicação entre os profissionais, garantindo que os benefícios desse vínculo ultrapassem a teoria e, de fato, beneficiem a população ${ }^{(24-25)}$.

A atuação interdisciplinar implica em reflexão-açãoreflexão ${ }^{(9)}$. Esse constante construir, desconstruir e reconstruir pode colaborar para a atuação dos profissionais que atuam junto a crianças e adolescentes com doenças crônicas, inclusive para aqueles que convivem com a asma.

A visão ampliada de profissionais das diferentes categorias podem contribuir para a avaliação e manejo da asma em crianças e adolescentes, especialmente nas orientações relativas à adesão ao tratamento, ao uso correto das medicações, controle ambiental de alérgenos e irritantes, identificação e estabelecimento de condutas relativas aos planos de ação durante as crises ou exacerbações da asma.

\section{CONCLUSÃO}

Há uma crescente divulgação de estudos voltados para a avaliação clínica e ambiental de crianças e adolescentes asmáticos. Essa tendência ocorre em virtude do impacto da doença crônica no cotidiano dos pacientes e seus familiares e, principalmente, da maior 
atenção dos profissionais de saúde. Além disso, aspectos epidemiológicos e sociais reforçam essa propensão. Entretanto, a atenção interdisciplinar no manejo da doença tem sido pouco explorada.

O ambulatório selecionado para realização deste estudo é referência no atendimento a crianças e adolescentes asmáticos e adota, como metodologia de trabalho, a atenção interdisciplinar. Trata-se de um modelo interessante, pois a condução das ações é feita com o intuito de promover a união dos profissionais em torno de objetivos comuns, o manejo adequado da asma e a promoção da saúde das crianças e adolescentes.

Neste estudo observou-se que, após acompanhamento multiprofissional e interdisciplinar, as crianças e adolescentes apresentaram melhora clínica da asma. Houve destaque para a redução dos sintomas, melhor nível de controle atual da doença e adesão ao uso contínuo de medicações específicas para o tratamento da asma. Portanto, a assistência qualificada parece favorecer o manejo da doença, inferindo-se que a intervenção da equipe multiprofissional impactou de forma positiva.

\section{REFERÊNCIAS}

1. Sociedade Brasileira de Pneumologia e Tisiologia. V Diretrizes brasileiras para o manejo da asma. J Bras Pneumol. 2012;38:01-46.

2. Global Initiative for Asthma - GINA [Internet]. Diagnosis and management of Asthma in children 5 years and younger [acesso em: 09 de abr 2015] Pocket guide for management and prevention, 2014. Disponível em:

http://www.ginasthma.org/local/uploads/files/GINA_Pocket_2 014_Jun11.pdf.

3. Global Initiative for Asthma - GINA [Internet]. Bethesda: global initiative for asthma [acesso em: 10 de fev 2014] Global strategy for asthma management and prevention, 2010. Disponível em:

http://www.ginasthma.org/pdf/GINA_Report_2010.pdf. 4. DATASUS [Internet]. Brasília: Ministério da saúde (BR) [cited 2012 jun 10]. Informações de saúde. Dados sobre morbidade hospitalar do SUS - por local de internação - DATASUS [Internet]. Disponível em:

http://tabnet.datasus.gov.br/cgi/tabcgi.exe?sih/cnv/miuf.def.

5. Amaral LM, Palma PV, Leite ICG. Evolução das políticas públicas e programas de controle da asma no Brasil sob a
O estabelecimento de novas estratégias de assistência qualificada às crianças e adolescentes asmáticos deve se dar em todos os níveis de atenção à saúde, especialmente, na atenção básica. As ações voltadas para o manejo adequado da doença nesse nível de atenção podem colaborar efetivamente para a redução de prejuízos para a saúde infantil, diminuição da sobrecarga de atendimentos nos serviços secundários e terciários, bem como, redução de custos relacionados aos atendimentos de emergência e internações por asma.

Dada a complexidade dos fatores envolvidos no tratamento da criança e do adolescente com asma, a formação de uma equipe multiprofissional, que atue de forma integrada, pode ser fundamental para assegurar a atenção adequada a essa população. O desenvolvimento de atividades interdisciplinares pode contribuir para a superação da fragmentação do cuidado, favorecer o comprometimento dos profissionais de saúde e promover a qualidade do serviço especializado, possibilitando um escopo mais ampliado, que contemple não apenas as necessidades clínicas dos pacientes, mas também aspectos sociais e educacionais.

perspectiva dos consensos. J Bras Pneumol. 2012;38(4):51825.

6. Stelmach R, Neto AC, Fonseca ACCF, Ponte EV, Alves G, Araujo-Costa In, et al. A workshop on asthma management programs and centers in Brazil: reviewing and explaining concepts. J Bras Pneumol. 2015;41(1):03-15.

7. Wall-Haas CL, Kulbok P, Kirchgessner J, Rovnyak V. Shared medical appointments: facilitating care for children with asthma and their caregivers. J Pediatr Health Care.

2012;26(1):37-44.

8. Yilmaz O, Eroglu N, Ozalp D, Yuksel H. Beliefs about medications in asthmatic children presenting to emergency department and their parents. J Asthma. 2012;49(3):282-7. 9. Meireles BHS, Erdmann AL. A interdisciplinaridade como construção do conhecimento em saúde e enfermagem. Texto $\&$ contexto enferm. 2005;14(3):411-8.

10. Ministério da Saúde; Conselho Nacional de Saúde. Resolução № 196/96 - Normas regulamentadoras de pesquisa envolvendo seres humanos. Brasília (Brasil): Ministério da Saúde; 1996.

11. Nóbrega VM, Reichert APS, Silva KL, Coutinho SED, Collet N. Imposições e conflitos no cotidiano das famílias de crianças 
com doença crônica. Esc. Anna Nery Rev. Enferm.

2012;16(4):781-8.

12. Simões SM, Cunha SS, Barreto ML, Cruz AA. Distribution of severity of asthma in childhood. J Pediatr. 2010; 86(5):417-23. 13. Zillmer LR, Gazzotti MR, Nascimento OA, Montealegre F, Fish J, Jardim JR. Gender differences in the perception of asthma and respiratory symptoms in a population sample of asthma patients in four Brazilian cities. J Bras Pneumol. 2014;40(6):591-8.

14. Bisgaard $\mathrm{H}$, Bonnelykke K. Long-term studies of the natural history of asthma in childhood. J Allergy Clin Immunol. 2010;126(2):187-97.

15. LeBlanc A, Robichaud P, Lacasse Y, Boulet LP. Quantification of asthma control: validation of the Asthma Control Scoring System. Allergy. 2007;62(2):120-5.

16. Nathan RA, Sorkness CA, Kosinski M, Schatz M, Li JT, Marcus $P$, et al. Development of the asthma control test: a survey for assessing asthma control. J Allergy Clin Immunol. 2004;113(1):59-65.

17. Amaral LM, Palma PV, Leite ICG. Considerações sobre a asma de interesse para a atenção primária: epidemiologia, impacto econômico e políticas públicas. Rev APS.

2012;15(4):508-16.

18. Bush A, Fleming L. Diagnosis and management of asthma in children BMJ. 2015;350:h996.

19. Mendes MA, Sant'Anna CC, March MFBP. Stress in children and adolescents with asthma. Rev. bras. crescimento desenvolv. hum. 2013; 23(1): 80-6.

20. Goodwin RD, Robinson M, Sly PD, McKeague IW, Susser $E S$, Zubrick SR, et al. Severity and persistence of asthma and mental health: a birth cohort study. Psychol Med. 2013;43(6):1313-22.

21. Souza MLM, Meneghini AC, Vianna EOB, Carvalho M. Técnica e compreensão do uso dos dispositivos inalatórios em pacientes com asma ou DPOC. J Bras Pneumol. 2009;35(9):82431. 22. Olin JT, Wechsler ME. Asthma: pathogenesis and novel drugs for treatment. BMJ. 2014;349:5517.

23. Camargo JSO, Magalhães PB, Fernandes ICF, Ramalho WS, Costa LDC, Salge AKM, et al. Utilização e eficácia de espaçadores no tratamento farmacológico de pacientes asmáticos: uma revisão integrativa. Rev. enferm. UERJ. 2012;20(1):654-60.

24. Costa RP. Interdisciplinaridade e equipes de saúde: concepções. Mental. 2007;5(8):107-24.

25. Menossi MJ, Lima RAG, Corrêa AK. A dor e o desafio da interdisciplinaridade no cuidado à criança. Rev. latinoam. enferm. 2008;16(3):489-94.

Recebido: 26/05/2014.

Aceito: 20/03/2015.

Publicado: 30/09/2015. 